\title{
Editorial
}

\section{Advanced Nanoporous Materials: Synthesis, Properties, and Applications}

\author{
Sayan Bhattacharyya, ${ }^{1}$ Yitzhak Mastai, ${ }^{2}$ Rabi Narayan Panda, \\ Sun-Hwa Yeon, ${ }^{4}$ and Michael Z. $\mathrm{Hu}^{5}$ \\ ${ }^{1}$ Department of Chemical Sciences, Indian Institute of Science Education and Research (IISER) Kolkata, Mohanpur 741246, India \\ ${ }^{2}$ Department of Chemistry and the Institute of Nanotechnology, Bar-Ilan University, 52900 Ramat Gan, Israel \\ ${ }^{3}$ Department of Chemistry, Birla Institute of Technology and Science Pilani, K K Birla Goa Campus, Zuari Nagar, \\ Goa 403726, India \\ ${ }^{4}$ Distributed Power Generation and Energy Storage Group, Korea Institute of Energy Research, Daejeon 305-343, Republic of Korea \\ ${ }^{5}$ Energy and Transportation Science Division, Oak Ridge National Laboratory, Oak Ridge, TN 37831-6181, USA \\ Correspondence should be addressed to Sayan Bhattacharyya; sayanb@iiserkol.ac.in
}

Received 12 June 2014; Accepted 12 June 2014; Published 19 June 2014

Copyright (C) 2014 Sayan Bhattacharyya et al. This is an open access article distributed under the Creative Commons Attribution License, which permits unrestricted use, distribution, and reproduction in any medium, provided the original work is properly cited.

Nanoporous materials are well known to be technologically useful for a wide spectrum of applications such as energy storage and conversion in fuel cells, solar cells, Liion batteries, hydrogen storage and supercapacitors, catalysis, sorption applications, gas purification, separation technologies, drug delivery, cell biology, environmental remediation, water desalination, purification, separation, sensors, optical, and electronic and magnetic devices. Typical examples of natural and synthetic nanoporous solids are zeolites, activated carbon, metal-organic frameworks, covalent organic frameworks, ceramics, silicates, nonsiliceous materials, aerogels, pillared materials, various polymers, and inorganic porous hybrid materials. However, the applicability of the porous nanomaterials depends on their targeted design at the atomic and molecular level which controls their porosity and surface area. The nanoporous materials can be synthesized in the laboratories using organic or inorganic templates. The self-assembly of organic templates or the existing pore size of the inorganic templates controls the porosity of the final product. In addition, the nanometer-size pores can be utilized to impregnate nanoparticles/proteins/ions to create multifunctional hybrids of practical and scientific interests. Considering the widespread applications of these nanoporous materials, uncovering their recent synthesis approaches, structure-dependent properties and potential applications in various disciplines of science and engineering are necessary and urgent.

This special issue focuses on the recent advances in synthesis approaches, newer properties, and environmental applications of nanoporous materials. A total of 6 research articles reporting on the synthetic design, characterization, property, analysis, and applications are presented in this special issue. The articles cover diverse nanoporous materials and morphologies such as anodic $\mathrm{CaO}-\mathrm{TiO}_{2}$ nanotubes (C. W. Lai), novel silica nanowires with intrawire lamellae (M. Z. $\mathrm{Hu}$ et al.), Fe nanoparticles (S.-H. Chaung et al.), montmorillonite with $7-9 \mathrm{~nm}$ pores (Y. Li et al.), chitosan polymer membrane (Y.-J. Chuang et al.), and a model SiC with C-terminated surface (M. Calvino et al.). The preparation techniques such as rapid-anodic oxidation electrochemical anodization (C. W. Lai), evaporation-induced self-assembly inside 50-200 $\mathrm{nm}$ diameter channels of anodized aluminum oxide and polycarbonate (M. Z. Hu et al.), borohydride reduction (S.-H. Chaung et al.), ball milling (Y. Li et al.), and desolvation (Y.-J. Chuang et al,) have been extensively presented. The electronic properties of porous $\mathrm{SiC}$ were investigated by ab initio DFT method (M. Calvino et al.). Most importantly, these articles cover wide application prospects, 
namely, $\mathrm{CO}_{2}$ adsorption (C. W. Lai), sulfide removal from water and wastewater (S.-H. Chaung et al.), adsorption of zearalenone, mycotoxin found in cereals ( $\mathrm{Y}$. Li et al.), and in vitro sampling of biomolecules (Y.-J. Chuang et al.). All the 6 contributions represent significant advances in the modern research directions on nanoporous materials. We hope that this issue will further promote developments in the synthesis protocols, characterization techniques, property analysis, and applications of advanced nanoporous materials.

\title{
Acknowledgments
}

We would like to thank all the authors and coauthors for contributing their important research and all the reviewers for their valuable time and dedication to this special issue.

\author{
Sayan Bhattacharyya \\ Yitzhak Mastai \\ Rabi Narayan Panda \\ Sun-Hwa Yeon \\ Michael Z. Hu
}



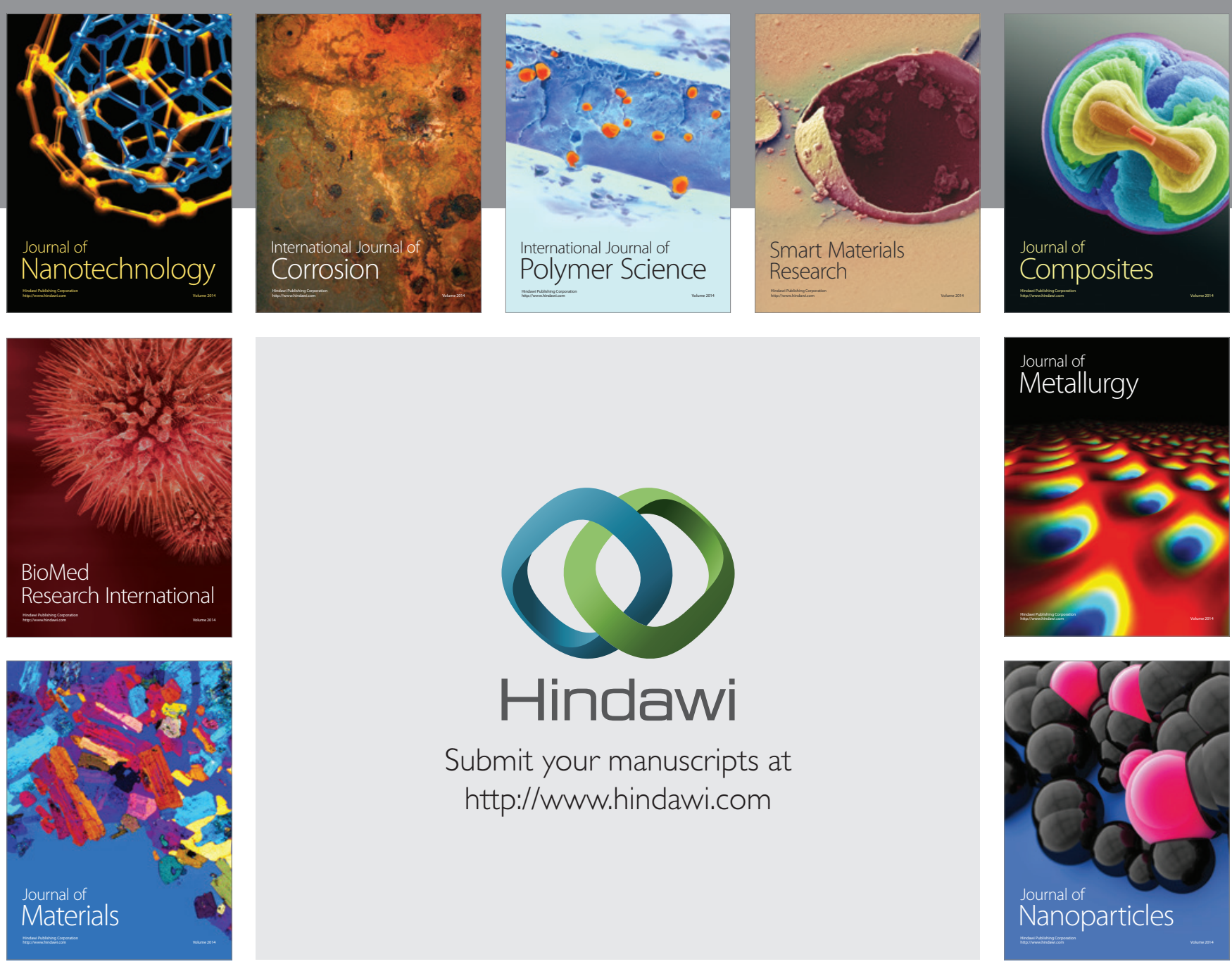

Submit your manuscripts at http://www.hindawi.com
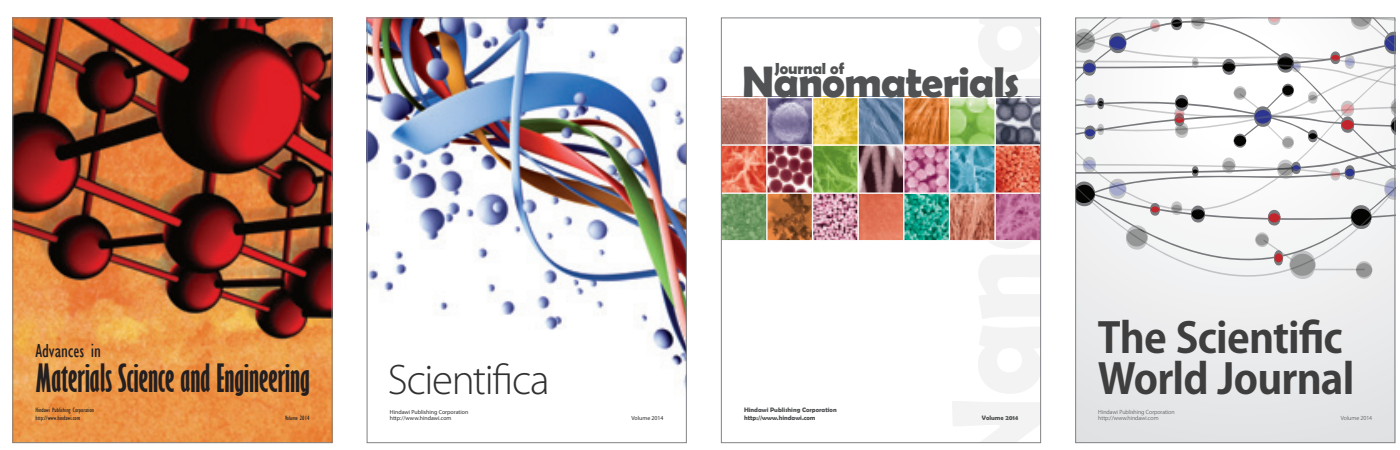

\section{The Scientific World Journal}
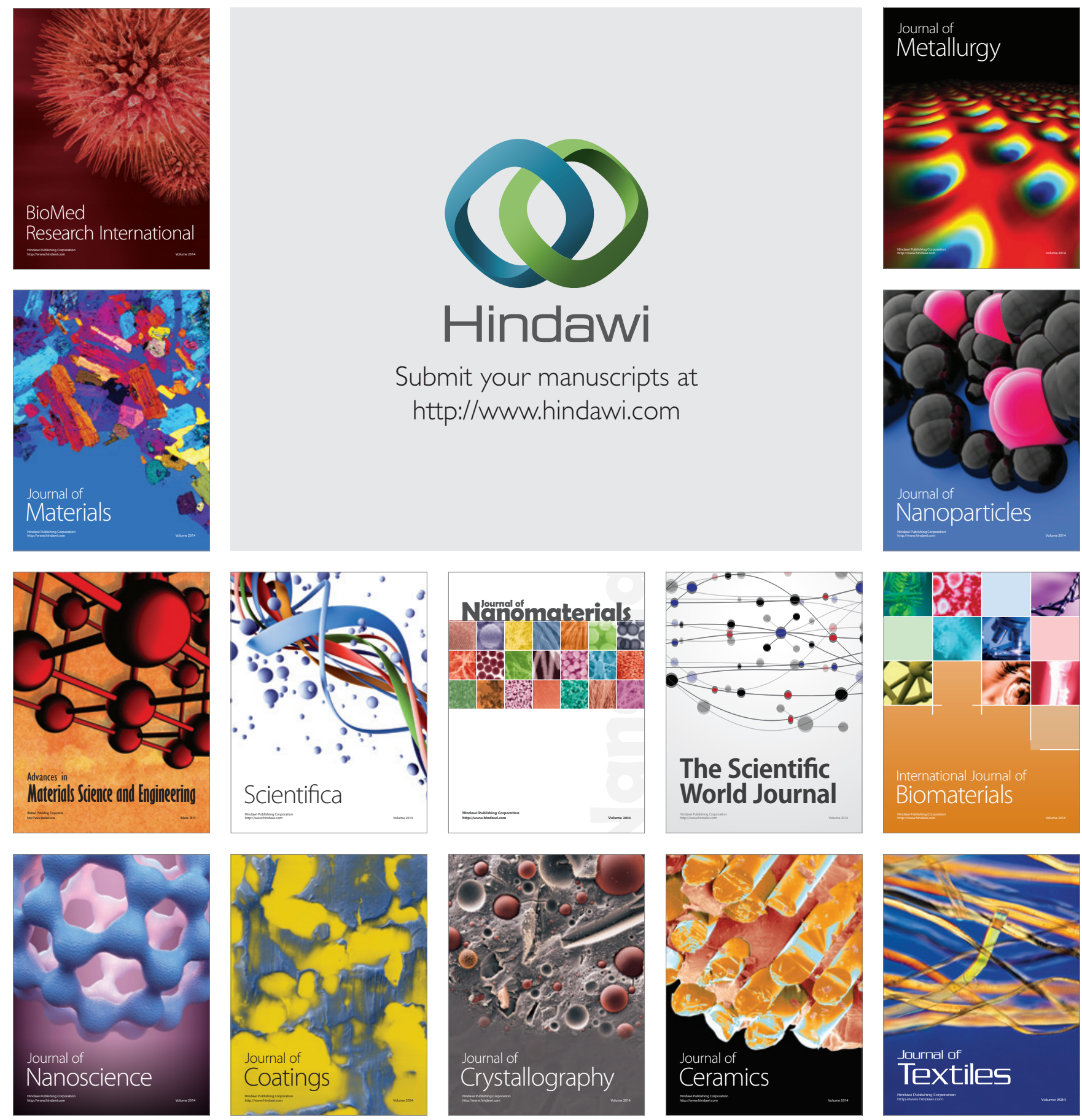